\title{
Coronavirus Disease 2019 and Pregnancy: What An Anesthesiologist Needs to Know
}

\author{
Poonam Kumari ${ }^{1}$, Amarjeet Kumar ${ }^{2}$, Chandni Sinha ${ }^{3}$, Ajeet Kumar ${ }^{4}$
}

\begin{abstract}
Coronavirus disease 2019 (COVID-19) is a pandemic, has emerged as a public health crisis for the entire world. In contrast to other areas of healthcare which can be delayed to priorities treatment of COVID-19 patients, the care of obstetric patients remains a clinical priority. All the patients and their support persons having suspected consistent symptoms of COVID-19 should be completed the screening via phone or video conferencing. The CDC has issued notifications that labor and delivery floors be identified for those pregnant patients with known or suspected positive cases and allow the staff to make the proper arrangements for isolation rooms and personal protective equipment (PPE). Due to uncertainty about the severity of COVID-19 and no clear evidence of vertical transmission, obstetric care is directed toward social distancing as a protective mechanism, and mother/baby separation to avoid contact transmission.
\end{abstract}

Keywords: Coronavirus, Personal protective equipment, Pregnancy.

Panamerican Journal of Trauma, Critical Care \& Emergency Surgery (2021): 10.5005/jp-journals-10030-1313

Coronavirus disease 2019 (COVID-19) is a pandemic, has emerged as a public health crisis for the entire world. In contrast to other areas of healthcare which can be delayed to prioritize treatment of COVID-19 patients, the care of obstetric patients remains a clinical priority. ${ }^{1}$ Symptoms to screen in COVID-19 obstetric patient include a history of fever, cough, shortness of breath, diarrhea, and history of possible exposure to COVID-19. However, pregnancy symptoms may overlap with mild COVID-19 disease and many patients may be asymptomatic at the time of admission. Screening pregnant patients may miss infection with SARS-CoV-2 in communities with a high prevalence or high projected infection rate. $^{2}$

Procedures like preoperative screening, cesarean deliveries, labor analgesia, and elective labor inductions must be ready for all pregnant patients. All patients and supports persons with consistent symptoms of COVID-19 should be screened via telephone or video conferencing. ${ }^{3}$ The $C D C$ has issued notifications that separate labor and delivery floors must be designated for those pregnant patients with known or suspected positive case, allowing the hospital staff to make the proper arrangements for isolation rooms and personal protective equipment (PPE). ${ }^{2}$ Before any procedure, it must be ensured that the number of healthcare workers is reduced to a minimum necessary, and specified in the delivery room or operation theater. All healthcare works involved in the obstetric procedures must be well trained on donning and doffing proper PPE, including face shield protection, N95 mask, or powered air-purifying respirator (PAPR) for aerosolizing procedures. The patient must wear a surgical mask throughout the procedure, during transportation, and during the postoperative period to minimize the risk of infections. Before any procedure, the operation theater or labor room should be kept ready, including all equipment, all emergency drugs, and medications for labor analgesia, cesarean delivery, and general anesthesia. If possible, positive or suspected positive patients should be confined to a negative pressure room. Visitors must be limited to one support person, while the use of video messaging with other ways of distant communications between the patient and families should
${ }^{1,3,4}$ Department of Anesthesiology, All India Institute of Medical Sciences, Patna, Bihar, India

${ }^{2}$ Department of Trauma and Emergency, All India Institute of Medical Sciences, Patna, Bihar, India

Corresponding Author: Amarjeet Kumar, Department of Trauma and Emergency, All India Institute of Medical Sciences, Patna, Bihar, India, Phone: +91 9570890646, e-mail: amarjeetdmch@gmail.com

How to cite this article: Kumari P, Kumar A, Sinha C, et al. Coronavirus Disease 2019 and Pregnancy: What An Anesthesiologist Needs to Know. Panam J Trauma Crit Care Emerg Surg 2021;10(1):51-52.

Source of support: Nil

Conflict of interest: None

be promoted. Neuraxial anesthesia is suggested in obstetric anesthesia and mainly in women with COVID-19. It is used to minimize the risk of infection from aerosolization associated with general anesthesia. Although high-efficiency particulate air (HEPA) filter is commonly used, in general anesthesia, it must be kept ready at all times in anticipation of neuraxial anesthesia failure and need to change to general anesthesia. Video-laryngoscopy is recommended to minimize mask ventilation. The later should be avoided due to the potential aerosolization, and patients must be extubated to oxygen mask with flows or nasal cannulas. All above procedures should be performed by experienced anesthetist with the use of proper PPE. The summary of obstetric anesthesia care is shown in Table 1.

The standard setting for appropriate care of a healthy and term newborn is the same room as mothers; commonly called "rooming-in". Current evidence suggests that the risk of a neonate acquiring SARS-CoV-2 from the mother is low. Furthermore, data suggest that there is no difference in risk of SARS-CoV-2 infection to the neonate, whether the neonate is cared for in a separate room or remains in the mother's room. ${ }^{4}$ Mothers should wear a mask and practice hand hygiene when in contact with the neonates. Mothers who room-in with their infants can more easily learn and 


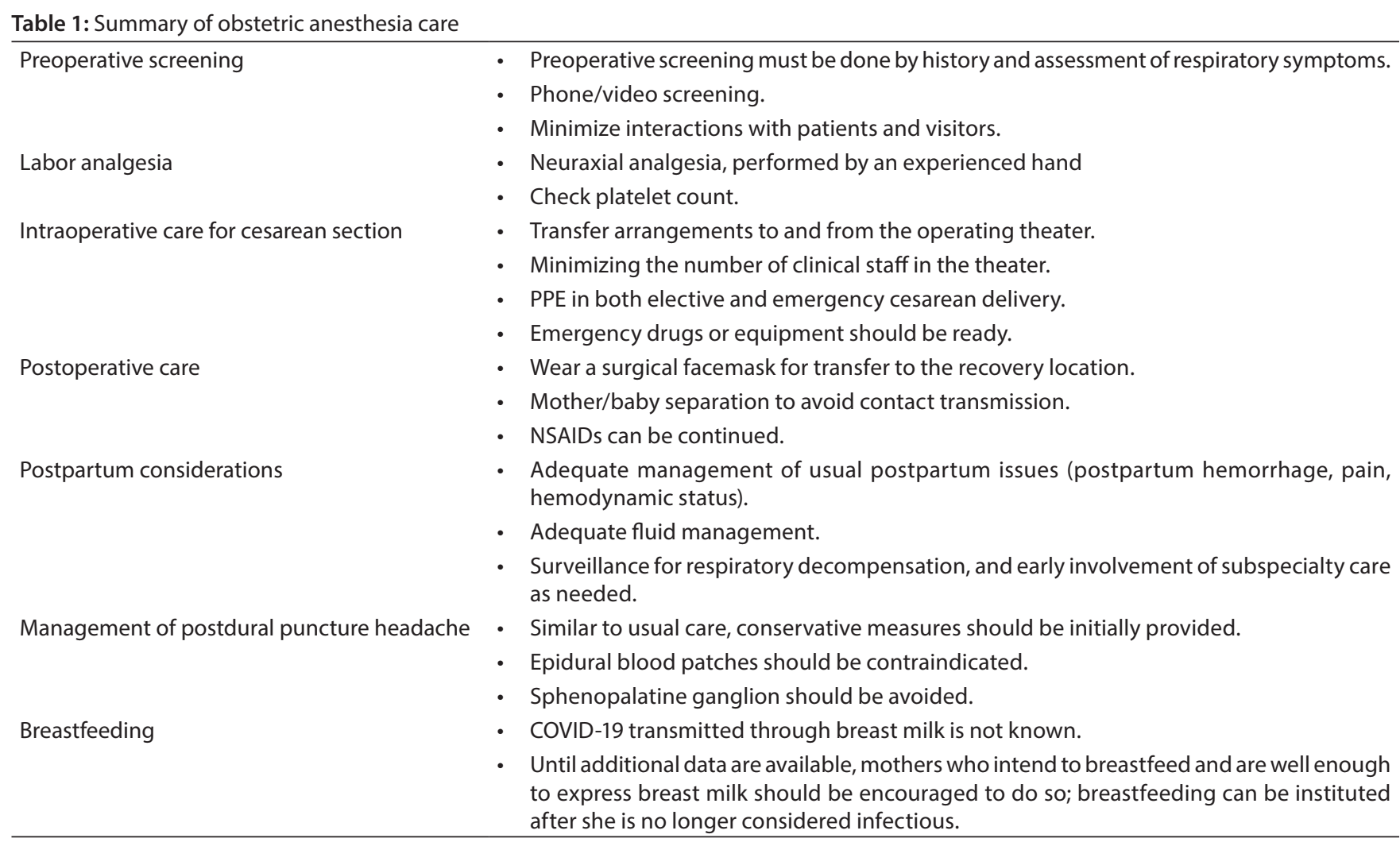

respond to their feeding cues, which supports to the establishment of breastfeeding. Breastfeeding reduces morbidity and mortality for both mothers and their infants. ${ }^{5}$

After surgery, an infected or possibly infected patient is to be observed in the operating room until discharge to the isolation ward, or room. Operation theater and the shifting route to the ward or room should be well disinfected. A collective effort between anesthesiologists, obstetricians, neonatologists, nursing, infectious disease physicians, and environmental services is required to minimize infection risks to patients as well as to the healthcare workers.

\section{References}

1. England $N$, Critical care and anesthesia service reorganisation 2020. Availablefrom: https://www.england.nhs.uk/coronavirus/
wpcontent/uploads/sites/52/2020/03/Specialty-guide_Critical-careand-anaesthesia-service-reorganisation_V1_17-March.pdf.

2. Centers for Disease Control and Prevention (CDC): Considerations for Inpatient Obstetric Healthcare Settings. Available at: https://www. cdc.gov/coronavirus/2019-ncov/hcp/inpatient-obstetric healthcareguidance.html.

3. Society for Obstetric Anesthesia \& Perinatology (SOAP). Interim Considerations for Obstetric Anesthesia Care Related to COVID19. Available at: https://soap.org/education/provider-education/expertsummaries/interim-considerations-for obstetric-anesthesia-carerelated-to-covid19.

4. CDC care for newborns https://www.cdc.gov/coronavirus/2019-ncov/ hcp/caring-for-newborns.html.

5. CDC Care for Breastfeeding Women. Interim Guidance on Breastfeeding and Breast Milk Feeds in the Context of COVID-19 https://www.cdc.gov/coronavirus/2019-ncov/hcp/care-forbreastfeeding-women.html. 